\title{
Video Article \\ Caspase-3 Activity in the Rat Amygdala Measured by Spectrofluorometry After Myocardial Infarction
}

\author{
Kim Gilbert ${ }^{1}$, Roger Godbout ${ }^{1}$, Guy Rousseau ${ }^{1}$ \\ ${ }^{1}$ Centre de Recherche, Hospital du Sacre-Coeur de Montreal \\ Correspondence to: Guy Rousseau at Guy.Rousseau@umontreal.ca \\ URL: https://www.jove.com/video/53207 \\ DOI: doi:10.3791/53207
}

Keywords: Neuroscience, Issue 107, Myocardial infarction, Depression, Caspase-3, Limbic system, Amygdala, Apoptosis

Date Published: 1/12/2016

Citation: Gilbert, K., Godbout, R., Rousseau, G. Caspase-3 Activity in the Rat Amygdala Measured by Spectrofluorometry After Myocardial Infarction. J. Vis. Exp. (107), e53207, doi:10.3791/53207 (2016).

\section{Abstract}

Myocardial infarction (MI) has dramatic mid- and long-term consequences at the physiological and behavioral levels, but the mechanisms involved are still unclear. Our laboratory has developed a rat model of post-MI syndrome that displays impaired cardiac functions, neuronal loss in the limbic system, cognitive deficits and behavioral signs of depression. At the neuronal level, caspase-3 activation mediates post-MI apoptosis in different limbic regions, such as the amygdala - peaking at 3 days post-MI. Cognitive and behavioral impairments appear 2-3 weeks post-MI and these correlate statistically with measures of caspase- 3 activity. The protocol described here is used to induce MI, collect amygdala tissue and measure caspase- 3 activity using spectrofluorometry. To induce MI, the descending coronary artery is occluded for 40 $\mathrm{min}$. The protocol for evaluation of caspase- 3 activation starts 3 days after Ml: the rats are sacrificed and the amygdala isolated rapidly from the brain. Samples are quickly frozen in liquid nitrogen and kept at $-80{ }^{\circ} \mathrm{C}$ until actual analysis. The technique performed to assess caspase-3 activation is based on cleavage of a substrate (DEVD-AMC) by caspase-3, which releases a fluorogenic compound that can be measured by spectrofluorometry. The methodology is quantitative and reproducible but the equipment required is expensive and the procedure for quantifying the samples is time-consuming. This technique can be applied to other tissues, such as the heart and kidneys. DEVD-AMC can be replaced by other substrates to measure the activity of other caspases.

\section{Video Link}

The video component of this article can be found at https://www.jove.com/video/53207/

\section{Introduction}

Caspases or Cysteine-dependent aspartate-directed proteases are a family of enzymes that are involved in various homeostasis processess ${ }^{1}$. Caspases can be broadly classified according to their roles in apoptosis or inflammation. Caspase-1, $-4,-5$ and -12 are involved in inflammation whereas those engaged in apoptosis can be sub-classified as initiator caspases (caspase- 8 and -9 ) and executioner caspases (caspase-3, -6 and -7 ).

Caspases play significant roles in many pathologies, mainly in disorders where apoptosis can be excessive, as observed after myocardial infarction (MI) or cerebral ischemia.

In the rat model of post-MI syndrome used in our lab, it is established that caspase- 3 is activated not only in the myocardium ${ }^{2}$ but also in the limbic system which is implicated in the control of mood and emotions ${ }^{3-6}$. It is also seen that caspase- 3 is activated in different regions of the limbic system, such as the amygdala and hippocampus, and this activation peaks around 3 days after ischemic insult ${ }^{4}$. Interestingly, caspase- 3 activity correlates with post-MI behavioral impairments, and its attenuation through pharmacological and nutritional interventions reduces these injuries, suggesting a possible link between caspase-3 and post-MI depression ${ }^{7-12}$.

Caspase- 3 activation can be measured by various techniques. Western blotting ascertains the enzymatic properties of the caspases and can detect active enzymes as well as their pro-enzyme forms. Western blotting, however, is semi-quantitative, and small variations can be lost through weak signal-to-noise ratios ${ }^{13}$. A better technique is based on the cleavage of a substrate (DEVD-AMC) by caspase-3 that releases a fluorogenic compound and can be measured by spectrofluorometry. Caspase-3 activation is a reliable marker of apoptosis. Measurement of apoptosis can be unstable because the time window of occurrence is short, and neighboring cells could phagocytize apoptotic bodies or cells ${ }^{14}$. Apoptosis can be further confirmed by other techniques, such as Terminal deoxynucleotidyl transferase dUTP nick end labeling (TUNEL) assay, that detects DNA fragmentation resulting from apoptotic signaling cascades ${ }^{6}$, or the ratio of pro-/anti-apoptotic proteins, such as Bax/Bcl2 ${ }^{6}$.

\section{Protocol}

Animals are handled in compliance with regulation of the local Animal Care Committee, in accordance with the Guidelines of the Canadian Council on Animal Care. Rats are housed individually under constant conditions (temperature of $21-22^{\circ} \mathrm{C}$ and humidity of $40-50 \%$ ), including a 12 hr dark- 
light cycle, beginning at 08:00 am. Chow pellets and tap water are available ad libitum throughout the study. An acclimatisation period of 3 days after delivery by the supplier is allowed before the experiment. Male Sprague-Dawley rats weighing between $300-800 \mathrm{~g}$ have been routinely used with this protocol.

\section{MI Surgery}

1. Induce anesthesia with intraperitoneal injections of ketamine $60 \mathrm{mg} / \mathrm{kg}$ and xylazine $10 \mathrm{mg} / \mathrm{kg}$. Confirm proper anesthetization by absence of reflex when the paws are pinched.

2. Shave surgical site. Intubate rat with endotracheal tubing: catheter (16G 1.77 inches) and place animal in lateral decubitus position. Place the animals on a heating pad to maintain temperature around $37^{\circ} \mathrm{C}$. Connect the endotracheal tubing to isoflurane $2 \%$.

3. Prepare surgical site with chlorhexidine gluconate and isopropyl alcohol. During surgery, use sterile gloves and sterilized instruments. Apply ophthalmic ointment to both eyes to prevent dryness while under anesthesia. Put sterile field on animal and the instruments on another sterile field next to the animal.

4. Incise skin with scalpel blade \#10. Peel off muscle tissue with a hemostatic clamp. Place the animal in ventral decubitus position. Open thoracic wall with a hemostatic clamp and position chest retractor. Open pericardium with a hemostatic clamp.

5. To produce coronary occlusion, loop a $360 \mathrm{~mm}$-long 4-0 silk suture around the descending coronary artery, passing through the contiguous myocardial tissue. Insert both ends of the silk suture into a $14 \mathrm{G}, 1.25 \mathrm{~cm}$ long plastic tube.

6. Pull both ends of the silk suture and push the tube down against the artery to occlude it, for 40 min. Secure occlusion by clamping the plastic tube with an hemostatic clamp.

7. After $40 \mathrm{~min}$, release the occlusion by releasing the hemostatic clamp, followed by removing the flexible tubing and silk suture. Note: Sham control rats undergo the same surgical procedure minus the occlusion of the coronary artery.

8. Before closing the thoracic wall, place a flexible catheter $(18 \mathrm{G}, 4.5 \mathrm{~cm})$ through the thoracic cavity to draw air out of the thorax with a $5-\mathrm{ml}$ syringe to prevent pneumothorax.

9. Close thorax cavity with 2-0 suture, stitch the muscle with 4-0 silk suture and the skin with 3-0 silk suture.

10. Before closing the last skin point, again draw air out of the thorax with 5-ml syringe through the catheter placed previously in the thorax cavity.

11. Suture last skin point. Stop isoflurane ventilation.

12. Monitor rat awakening. Do not leave an animal unattended until it has regained sufficient consciousness to maintain sternal recumbency.

13. Treat rat every $8 \mathrm{hr}$ for $24 \mathrm{hr}$ with an analgesic (buprenorphine, $0.05 \mathrm{mg} / \mathrm{kg}$ i.p.) and with an antibiotic (a single dose of duplocillin, $0.2 \mathrm{mg} / \mathrm{kg}$ i.p.).

14. After the surgery, house rats individually until time of sacrifice, i.e., 3 days post-MI.

15. Infarct size, mainly necrosis, is expressed as the proportion of infarcted tissue in the area at risk of the left ventricle. This can measured using triphenyltetrazolium coloration ${ }^{11}$.

\section{Amygdala Isolation}

1. Place the animal head first in a cone-shaped bag, with its nose protruding out of the narrow end aperture. Secure rat to avoid any movement. Position its head appropriately on the guillotine, between the scissor blades. Decapitate animal.

2. Place head of the rat in a dish kept on crushed ice. Perform all procedures for tissue isolation on crushed ice $\left(4^{\circ} \mathrm{C}\right)$.

3. Cut open skull with scissors, carefully detach bone sheaths with bone rongeurs by pulling up and avoiding mutilating the tissue underneath.

4. Place the flat blade of a spatula between the bottom of the skull and the posterior ventral surface of the brain and carefully detach brain from skull by gently pushing the blade forward along the bottom of the skull until the brain can be slowly lifted up out of the skull. Discard the skull.

5. Place brain on its dorsal surface. Identify hypothalamus (structure in front of the cerebellum) and cut the brain coronally in front of its anterior end and behind its posterior end; discard the anterior and posterior parts of the brain if not needed for other uses.

6. Visualize the amygdalas as small spheres underneath the temporal lobes, bilaterally, just next to the hypothalamus.

7. Flip the brain flat on its frontal end, dorsal surface away from the experimenter.

8. Start with left or right side of the brain. Separate the hemispheres and then the cortex from the contiguous amygdala. Cut way about 8 mm of this loose cortex to allow isolation of the amygdala. Cut away amygdala with scalpel.

9. Isolate the basolateral part of the amygdala from its centromedial part by cutting along the dark suture that runs across it, almost half and half. This structure is not always easily identifiable.

10. Put amygdala subparts in separate identified vials and keep on crushed ice.

11. Repeat dissection procedure with the other hemisphere.

12. Immerse vials in liquid nitrogen for $1 \mathrm{~min}$. Keep vial in $-80^{\circ} \mathrm{C}$ freezer until needed.

\section{Caspase-3 Activity}

1. Prepare lysis buffer with $0.32 \mathrm{M}$ of sucrose, $1 \%$ Triton-X-100, $10 \mathrm{mM}$ of Tris- $\mathrm{HCl}, \mathrm{pH} 8,5 \mathrm{mM}$ of ethylenediaminetetraacetic acid, $2 \mathrm{mM}$ of dithiothreitol, $1 \mathrm{mM}$ of phenylmethylsulfonyl fluoride, $10 \mu \mathrm{g} / \mu \mathrm{l}$ of leupeptin, $10 \mu \mathrm{g} / \mu \mathrm{l}$ of pepstatin $\mathrm{A}$ and $10 \mu \mathrm{g} / \mu \mathrm{l}$ of aprotinin.

2. Add $150 \mu \mathrm{l}$ of lysis buffer to each sample $(5-10 \mathrm{mg})$. Keep on ice. Sonicate each sample on ice for 5 sec at maximal intensity (40 W). Incubate tissue in lysis buffer on ice for $30 \mathrm{~min}$. Vortex each sample for $5 \mathrm{~min}$.

3. Perform 3 freeze/thaw cycles by placing samples alternately in liquid nitrogen and on a thermostatically-controlled heating plate set at $37^{\circ} \mathrm{C}$. Centrifuge tissue at $13,000 \mathrm{~g}\left(4^{\circ} \mathrm{C}\right)$ for $10 \mathrm{~min}$. Carefully remove supernatant and keep in tube on ice.

4. Prepare standard protein concentrations between 0 and $10 \mu \mathrm{g} / \mathrm{ml}$ with bovine serum albumin. Prepare blanks with buffer only.

5. Add $200 \mu \mathrm{l}$ of Bradford reagent to each standard. Read standard at $595 \mathrm{~nm}$. For protein levels, add $5 \mu \mathrm{l}$ of sample supernatant to $795 \mu \mathrm{l}$ of distilled water and $200 \mu \mathrm{l}$ of Bradford reagent. Read each sample at $595 \mathrm{~nm}$ and quantify protein with standard curve.

6. Prepare reaction buffer with $50 \mathrm{mM}$ Tris- $\mathrm{HCl}, \mathrm{pH} 7,5 \mathrm{mM}$ of $\mathrm{MgCl}_{2}, 1 \mathrm{mM}$ of ethylene glycol tetraacetic acid, $0.1 \%$ of 3cholamidopropyl)dimethylammonio]-1-propanesulfonate and $1 \mathrm{mM}$ of dithiothreitol.

7. Add $25 \mu \mathrm{g}$ of protein to reaction buffer and caspase-3 substrates to obtain positive and negative reactive samples. For negative samples, add reaction buffer, $25 \mu \mathrm{g}$ of protein, $0.4 \mu \mathrm{l}$ of Ac-DEVD-CHO $800 \mu \mathrm{M}$ and $0.8 \mu \mathrm{l}$ of Ac-DEVD-AMC $10 \mathrm{mM}$. For positive samples, add $25 \mu \mathrm{g}$ 
of protein to reaction buffer and $0.8 \mu \mathrm{l}$ of Ac-DEVD-AMC $10 \mathrm{mM}$. Process all negative and positive samples in triplicate. Complete the final volume of each sample up to $200 \mu$ by adding reaction buffer.

8. Produce negative controls by adding $188.7 \mu \mathrm{l}$ of reaction buffer to $0.5 \mu \mathrm{l}$ of Ac-DEVD-CHO $800 \mu \mathrm{M}, 0.8 \mu \mathrm{l}$ Ac-DEVD-AMC $10 \mathrm{mM}$ and $10 \mu \mathrm{l}$ of lysis buffer. For positive controls, add $189.2 \mu \mathrm{l}$ of reaction buffer, $0.8 \mu \mathrm{l}$ of Ac-DEVD-AMC $10 \mathrm{mM}$ and $10 \mu \mathrm{l}$ of lysis buffer.

9. Incubate samples and controls in the dark for $3 \mathrm{hr}$ at $37^{\circ} \mathrm{C}$.

10. Stop reaction with $600 \mu$ l glycine $0.4 \mathrm{M}$ and $\mathrm{NaOH} 0.4 \mathrm{M}(\mathrm{pH} \mathrm{10)}$ in each sample and control.

11. Quantify fluorescence by spectrofluorometry at excitation wavelength of $365 \mathrm{~nm}$ and emission wavelength of $465 \mathrm{~nm}$. Add $2 \mathrm{ml}$ of distilled water to reaction in glass cuvette. Read controls and samples for $10 \mathrm{sec}$ with 1 point at each sec.

12. Quantify specific activity in each sample: ((Fluorescence of negative samples minus fluorescence of positive samples) $x$ volume of $2.8 \mathrm{ml})$ divided by (time of incubation $3 \mathrm{hr} \times$ quantity of protein $25 \mathrm{mg}$ ).

\section{Representative Results}

The amygdala was isolated in 8 sham (control) and $8 \mathrm{Ml}$ rats three days after the induction of the ischemia/reperfusion protocol. Caspase-3 activity was measured in this tissue using a fluorochrome that can be cleaved by active caspase-3 and detected by spectrofluorometry. Positive and negative measurements (see steps 3.11, 3.12 and 3.16) were performed in triplicate and measured during $10 \mathrm{sec}$, with 1 point at each sec. Differences between positive and negative controls were calculated and the average of the differences for all sham tissue performed during this experiment was set at $100 \%$. Results from MI rats were scaled according to this reference and Figure 1 illustrates the final results: it indicates a significantly higher activity in MI rats compared to sham $(p<0.05)$.

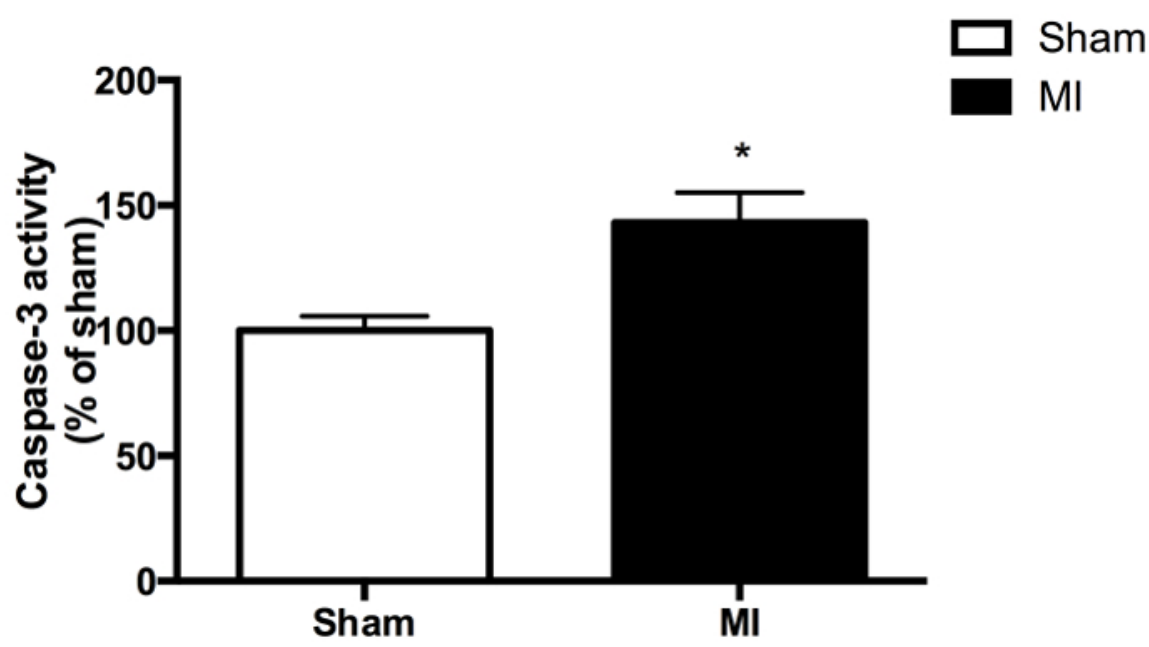

Figure 1. Caspase-3 activity in the amygdala of MI rats, expressed as percentage of mean activity in sham controls, set to $100 \%$ ( 8 rats per group). *Indicates significant between-group difference $(p<0.05)$. The data are expressed as mean \pm SEM.

\section{Discussion}

Our experience with this protocol over the last 10 years led to the identification of critical methodological issues. First, rapid brain dissection in a Petri dish on crushed ice is important to maintain preparation quality. Second, it must be realized that tissue sonication is shorter for the cerebral amygdala compared to other types of tissues, such as the heart $(10 \mathrm{sec})$ or kidneys $(20 \mathrm{sec})$. Third, great care should be taken to carefully mix the substrate before adding it to the samples. We encountered variable signals when the mixing sequence was inadequate. Fourth, no bubbles should be present in the quartz cell when reading the samples. Otherwise, the signal could be altered.

We made a change in the last few years to increase reproducibility of the results: increasing substrate volume to avoid volume less than $1 \mu \mathrm{l}$. Still, small variations may occur between successive assays, and at least 3 control samples should be used to control for variations. Fluorescence measures of these controls are averaged and the mean is set at $100 \%$. Fluorescence measures in experimental samples are transformed in percent of the control samples.

Another limitation of the technique is that only a portion of tissue is used and, therefore, caspase- 3 activity could be underestimated. Indeed, the time window of apoptosis is short in any given cell and could be missed even though it occurs in neighboring areas at the time of sampling $1,4,15$. The reverse is also possible: apoptosis could be particularly intense in portions of tissue, resulting in overestimation of caspase-3 activity. We recommend the use of remaining tissues (amygdala) for complementary techniques, such as TUNEL assays or Bax/Bcl2 ratio (see Introduction).

Spectrofluorometry has at least two advantages over Western blotting. First, it directly generates quantitative data. Second, it measures enzymatic activity itself rather than protein or RNA expression, which may be unrelated, and it is known that protein expression may be increased without any change in enzymatic activity. Moreover, spectrofluorometry can be performed on other tissues or with different animal species and can be adjusted for other caspase subtypes, with different substrates, so that safe comparisons can be made.

In conclusion, this document demonstrates the use of spectrofluorometry to measure caspase-3 activity in the amygdala, providing evidence that apoptosis occurs in this region of the limbic system 3 days after MI. 


\section{Disclosures}

The authors have nothing to disclose.

\section{Acknowledgements}

This work was supported by a grant from the Natural Sciences and Engineering Research Council of Canada. Kim Gilbert holds a studentship from Fonds de la recherche du Québec - Santé.

\section{References}

1. Denault, J. B., \& Salvesen, G. S. Caspases: keys in the ignition of cell death. Chemical Reviews. 102, 4489-4500, (2002).

2. Boucher, M. et al. Post-ischemic cardioprotection by A2A adenosine receptors: dependent of phosphatidylinositol 3-kinase pathway. Journal of Cardiovascular Pharmacology. 43, 416-422, (2004).

3. Kaloustian, S. et al. Celecoxib after the onset of reperfusion reduces apoptosis in the amygdala. Apoptosis. 12, 1945-1951, (2007).

4. Kaloustian, S. et al. Apoptosis time course in the limbic system after myocardial infarction in the rat. Brain Research. 1216, 87-91, (2008).

5. Rousseau, G., Bah, T. M., \& Godbout, R. Post-Myocardial Infarction Depression, Novel Strategies in Ischemic Heart Disease. (U. Lakshmanadoss, Ed.), Chapter 19, pp. 333-362, InTech (2012).

6. Wann, B. P. et al. Apoptosis detected in the amygdala following myocardial infarction in the rat. Biological Psychiatry. 59, 430-433, (2006).

7. Arseneault-Breard, J. et al. Combination of Lactobacillus helveticus R0052 and Bifidobacterium longum R0175 reduces post-myocardial infarction depression symptoms and restores intestinal permeability in a rat model. British Journal of Nutrition. 107, 1793-1799, (2012).

8. Bah, T. M. et al. Escitalopram reduces circulating pro-inflammatory cytokines and improves depressive behavior without affecting sleep in a rat model of post-cardiac infarct depression. Behavioural Brain Research. 225, 243-251, (2011).

9. Bah, T. M., Kaloustian, S., Rousseau, G., \& Godbout, R. Pretreatment with pentoxifylline has antidepressant-like effects in a rat model of acute myocardial infarction. Behavioural Pharmacology. 22, 779-784, (2011).

10. Gilbert, K. et al. Attenuation of post-myocardial infarction depression in rats by n-3 fatty acids or probiotics starting after the onset of reperfusion. British Journal of Nutrition. 109, 50-56, (2013).

11. Gilbert, K., Bernier, J., Godbout, R., \& Rousseau, G. Resolvin D1, a metabolite of omega-3 polyunsaturated fatty acid, decreases postmyocardial infarct depression. Marine Drugs. 12, 5396-5407, (2014).

12. Wann, B. P. et al. Behavioural signs of depression and apoptosis in the limbic system following myocardial infarction: effects of sertraline. Journal of Psychopharmacology (Oxford, England). 23, 451-459, (2009).

13. Gorr, T. A., \& Vogel, J. Western blotting re-visited: critical perusal of under-appreciated technical issues. Proteomics: Clinical Applications. (2015).

14. McKernan, D. P., Dinan, T. G., \& Cryan, J. F. 'Killing the blues': a role for cellular suicide (apoptosis) in depression and the antidepressant response? Progress in Neurobiology. 88, 246-263, (2009).

15. Green, D. R. Apoptotic pathways: paper wraps stone blunts scissors. Cell. 102, 1-4, (2000). 